Marquette University

e-Publications@Marquette

$11-2016$

\title{
Diffusion Tensor Imaging Predictors of Episodic Memory Decline in Healthy Elders at Genetic Risk for Alzheimer's Disease
}

\author{
Melissa A. Lancaster \\ Rosalind Franklin University of Medicine and Science \\ Michael Seidenberg \\ Rosalind Franklin University of Medicine and Science \\ J. Carson Smith \\ University of Maryland at College Park \\ Kristy A. Nielson \\ Marquette University, kristy.nielson@marquette.edu \\ John L. Woodard \\ Wayne State University
}

See next page for additional authors

Follow this and additional works at: https://epublications.marquette.edu/psych_fac

Part of the Psychology Commons

\section{Recommended Citation}

Lancaster, Melissa A.; Seidenberg, Michael; Smith, J. Carson; Nielson, Kristy A.; Woodard, John L.; Durgerian, Sally; and Rao, Stephen M., "Diffusion Tensor Imaging Predictors of Episodic Memory Decline in Healthy Elders at Genetic Risk for Alzheimer's Disease" (2016). Psychology Faculty Research and Publications. 231.

https://epublications.marquette.edu/psych_fac/231 


\section{Authors}

Melissa A. Lancaster, Michael Seidenberg, J. Carson Smith, Kristy A. Nielson, John L. Woodard, Sally Durgerian, and Stephen M. Rao 


\title{
Diffusion Tensor Imaging Predictors of Episodic Memory Decline in Healthy Elders at Genetic Risk for Alzheimer's Disease
}

\author{
Melissa A. Lancaster \\ Department of Psychology, \\ Rosalind Franklin University of Medicine and Science, \\ North Chicago, IL \\ Michael Seidenberg \\ Department of Psychology, \\ Rosalind Franklin University of Medicine and Science, \\ North Chicago, IL \\ Carson Smith \\ Department of Kinesiology, University of Maryland, \\ College Park, MD \\ Kristy A. Nielson \\ Department of Psychology, Marquette University, \\ Department of Neurology, Medical College of Wisconsin, \\ Milwaukee, WI
}


NOT THE PUBLISHED VERSION; this is the author's final, peer-reviewed manuscript. The published version may be accessed by following the link in the citation at the bottom of the page.

\author{
John L. Woodard \\ Department of Psychology, Wayne State University, \\ Detroit, $M I$ \\ Sally Durgerian \\ Department of Neurology, Medical College of Wisconsin, \\ Milwaukee, WI \\ Stephen M. Rao \\ Neurological Institute, Cleveland Clinic, \\ Cleveland, $\mathrm{OH}$
}

\begin{abstract}
Objectives: White matter (WM) integrity within the mesial temporal lobe (MTL) is important for episodic memory (EM) functioning. The current study investigated the ability of diffusion tensor imaging (DTI) in MTL WM tracts to predict 3-year changes in EM performance in healthy elders at disproportionately higher genetic risk for Alzheimer's disease (AD). Methods: Fifty-one cognitively intact elders ( $52 \%$ with family history (FH) of dementia and $33 \%$ possessing an Apolipoprotein E $\varepsilon 4$ allelle) were administered the Rey Auditory Verbal Learning Test (RAVLT) at study entry and at 3-year follow-up. DTI scanning, conducted at study entry, examined fractional anisotropy and mean, radial and axial diffusion within three MTL WM tracts: uncinate fasciculus (UNC), cingulate-hippocampal ( $\mathrm{CHG}$ ), and fornix-stria terminalis (FxS). Correlations were performed between residualized change scores computed from RAVLT trials 1-5, immediate recall, and delayed recall scores and baseline DTI measures; MTL gray matter (GM) and WM volumes; demographics; and AD genetic and metabolic risk factors. Results: Higher MTL mean and axial diffusivity at baseline significantly predicted 3-year changes in EM, whereas baseline MTL GM and WM volumes, FH, and metabolic risk factors did not. Both $\varepsilon 4$ status and DTI correlated with change in immediate recall. Conclusions: Longitudinal EM changes in cognitively intact, healthy elders can be predicted by disruption of the MTL WM microstructure. These results are derived from a sample with a disproportionately higher genetic risk for $A D$, suggesting that the observed WM disruption in MTL pathways may be related to early neuropathological changes associated with the preclinical stage of AD. (JINS, 2016, 22, 10051015)
\end{abstract}

\title{
Introduction
}

Episodic memory (EM) involves a widely distributed network that includes the mesial temporal lobe (MTL) as a central processing

Journal of the International Neuropsychological Society, Vol 22, No. 10 (November 2016): pg. 1005-1015. DOI. This article is (C) Cambridge University Press and permission has been granted for this version to appear in ePublications@Marquette. Cambridge University Press does not grant permission for this article to be further copied/distributed or hosted elsewhere without the express permission from Cambridge University Press. 
hub that communicates with the posterior cingulate, the diencephalic region, and cortical frontal lobe areas (Dickerson \& Eichenbaum, 2010; Mesulam, 1990). Subtle changes in EM are commonly associated with normal aging (Oishi, Faria, van Zijl, \& Mori 2011), but can also be the first and most prominent neuropsychological domain affected by Alzheimer's disease (AD) (Albert, Moss, Tanzi, \& Jones, 2001; Backman, Small, \& Fratiglioni, 2001; Bondi, Salmon, Galasko, Thomas, \& Thal, 1999). Successful EM requires efficient white matter (WM) connectivity, both within the MTL and between the MTL and other regions within the EM network.

Diffusion tensor imaging (DTI) provides a potentially important method for interrogating the integrity of MTL WM tracts that interconnect regions within the EM network. DTI studies have commonly found WM disruption in mild cognitive impairment (MCI) and $A D$ patients in limbic tracts important for $E M$, including the cingulate-hippocampal connections and the perforant pathway to the hippocampus (Canu et al., 2010; Salat et al., 2010; Zhang et al., 2007). The fornix, the major WM projection tract from the hippocampus to a diencephalic-frontal network, is also affected in MCI and AD (Bennett \& Madden, 2014; Geschwind, 1965a, 1965b; Wisse et al., 2015), as is the uncinate fasciculus, which connects the anterior temporal lobe to lateral orbitofrontal regions (Alves et al., 2012; Canu et al., 2010). Indeed, based on these DTI findings, some (Bartzokis et al., 2004) have suggested that AD pathology produces a "disconnection" between the network of regions subserving EM.

The purpose of the current longitudinal study was to determine if DTI measures are capable of predicting future EM changes in cognitively intact elders. We are aware of only one other such study. Zhuang et al. (2012) found lower baseline fractional anisotropy (FA) in the left parahippocampal cingulum, inferior temporal lobe, parahippocampal gyrus, and thalamus of cognitively intact, healthy elders who demonstrated verbal EM decline over the subsequent 2 years. Unlike the Zhuang et al. (2012) study, the current study recruited a disproportionately larger number of elders with a genetic risk of AD based on possession of an Apolipoprotein E (APOE) $\varepsilon 4$ allele and/or a family history $(\mathrm{FH})$ of dementia. This was done to increase the probability that some of our older participants would be in the preclinical stage of AD (Bondi et al., 1999). 
Change in EM was measured using the Rey Auditory Verbal Learning Test (RAVLT; Rey, 1958), which was administerd twice: at study entry and again at the 3-year follow-up examination. The RAVLT measures learning over trials as well as immediate and delayed recall. Whereas changes in both learning and immediate/delayed recall have been associated with healthy aging (Balthazar, Yasuda, Cendes, \& Damasceno, 2010; Vyhnalek et al., 2014), we hypothesized that WM abnormalities would be more sensitive in predicting changes in delayed recall performance, which has been shown to be a strong predictor of conversion from healthy aging to MCI or AD (Albert et al., 2001; Hamel et al., 2015; Mistridis, Krumm, Monsch, Berres, \& Taylor, 2015; Papp et al., 2015).

To minimize a potentially large number of statistical comparisons, we confined our DTI analysis to three MTL fiber tracts that are critical to EM function: the hippocampal projections of the cingulum, the fornix/stria terminalis, and the uncinate fasciculus. Left and right MTL structures were investigated separately based on findings of Zhuang et al (2012) that left but not right MTL structures predicted verbal EM decline. Additionally, left MTL structures correlate more strongly with verbal EM tasks in older adults than right MTL structures (Ezzati, Katz, Lipton, Zimmerman, \& Lipton, 2016; Rosen et al., 2003). Greater left MTL dysfunction is also observed in individuals with Alzheimer's disease (Loewenstein et al., 1989; Thompson et al., 2003).

In addition to FA, we also report results from three diffusion measures: mean diffusivity (MD), axial diffusivity (DA), and radial diffusivity (DR). Diffusion measures have been shown to be sensitive in distinguishing healthy aging from MCI and $A D$ (Acosta-Cabronero, Williams, Pengas, \& Nestor, 2010; Agosta et al., 2011; Nir et al., 2013). Whereas DA and DR are mathematically independent and are thought to reflect different types of WM pathology based on the direction of diffusion (Acosta-Cabronero et al., 2010; Song et al., 2003), MD is mathematically related to DA and DR, since MD represents an average of the two diffusion measures and reflects global alterations in diffusion rather than abnormalities in a specific diffusion direction. 
At study entry, we also recorded other imaging and non-imaging measures that could potentially predict EM decline in this sample of elders with a disproportionately higher genetic risk for AD. From anatomical scans, we measured volumes of key MTL structures associated with EM: hippocampus, entorhinal cortex, and parahippocampal gyrus. Longitudinal studies conducted in cognitively intact elders indicate that MRI volumes of the hippocampus and entorhinal cortex (Cardenas et al., 2011; den Heijer et al., 2006; Golomb et al., 1996; Woodard et al., 2010, 2012) predict EM decline in healthy elders. In addition, metabolic/vascular disorders are known preclinical risk factors for AD (Balthazar et al., 2010; Caselli et al., 2004). Thus, we also examined whether the presence/absence of metabolic/vascular disorders, that is, diabetes, hypertension, hypercholesterolemia, and elevated triglycerides, predict future EM decline.

The primary goal of this study was to test the hypothesis that DTI variables from MTL fiber tracts, collected at study entry, could predict 3-year change in EM test performance in elders with a preponderant genetic risk for AD. Our secondary aim examined whether DTI measures provide unique information regarding the prediction of future changes in EM performance compared to other well-established $A D$ risk factors, including possession of an APOE $\varepsilon 4$ allele, FH of dementia, volume of MTL WM and gray matter (GM) structures, and pre-existing metabolic/vascular disorders.

\section{Methods}

\section{Participants}

Fifty-one healthy, cognitively intact, older adults participated in a longitudinal study of genetic risk for Alzheimer's disease funded by the National Institute on Aging (R01 AG022304). Demographic characteristics of the sample (age, education, and sex) are shown in Table 1. Participants were initially recruited from newspaper advertisements and were excluded if they were currently taking psychoactive medication or had a history of neurologic, psychiatric, or medical conditions that may affect brain functioning. Details of the recruitment process and inclusion/exclusion criteria are provided in a 
previous publication (Seidenberg et al., 2009). The project was approved by the Medical College of Wisconsin Institutional Review Board; all participants provided written informed consent.

Table 1. Baseline demographic characteristics, $A D$ genetic risk factors, $A D$ metabolic risk factors, and neuropsychological testing.

\begin{tabular}{lrr}
\hline \hline & M (SD) & Range \\
\hline Demographic characteristics & & \\
Age - yr & $73.45(4.84)$ & $67-83$ \\
Education - yr & $14.40(2.22)$ & $11-20$ \\
Sex - $n$ (\%) females & $43(84.3)$ & \\
AD genetic risk factors & $17(33.3)$ & \\
APOE $84+-\mathrm{n}$ (\%) & $27(52.9)$ & \\
Family history - $\mathrm{n}(\%)$ & $14(27.4)$ & \\
AD metabolic risk factors & $16(31.3)$ & \\
Hypertension - $n$ (\%) & $6(11.7)$ & \\
Hypercholesterolemin - $n$ (\%) & & \\
Elevated triglycerides - $n$ (\%) & $29.5(0.90)$ & $25-30$ \\
Neuropsychological testing & $138.76(2.80)$ & $129-144$ \\
Mini Mental State Exam & $48.94(7.33)$ & $31-62$ \\
Mattis DRS-2 Total & $10.16(2.43)$ & $6-15$ \\
RAVLT Total Recall & $9.96(2.81)$ & $6-15$ \\
RAVLT Immediate Recall & \\
RAVLT Delayed Recall & \\
\hline \hline
\end{tabular}

Note. $A D=$ Alzheimer's disease; $A P O E=$ Apolipoprotein $E ;$ DRS-2=Dementia Rating Scale, 2nd Edition; RAVLT=Rey Auditory Verbal Learning Test.

To enrich the sample with individuals at genetic risk for $A D$, $52 \%(n=27)$ of the recruited sample had a FH of dementia (see Table 1). A positive $\mathrm{FH}$ was defined by either a clear clinical diagnosis of $A D$ or a reported history of gradual decline in memory and other cognitive functions in the absence of a formal diagnosis of $A D$ in a first-degree relative (i.e., parent or sibling). APOE genotype was then determined using a polymerase chain reaction method (Saunders et al., 1996); $33 \%(n=17)$ of the sample possessed one $(n=15 ; 29.4 \%)$ or both $(n=2 ; 3.9 \%)$ APOE $\varepsilon 4$ alleles.

Participants completed a health questionnaire regarding their history of potential metabolic risk factors for $A D$ (i.e., diabetes, hypertension, hypercholesterolemia, elevated triglycerides). Data were coded dichotomously (condition present/absent) and were based on 
participant self-report. Table 1 indicates the number of patients with each self-reported metabolic condition. Only one participant reported being treated for diabetes.

All participants were administered the following measures at study entry to ensure they were cognitively intact: Mini Mental State Examination (MMSE; Folstein, Folstein, \& McHugh, 1975), RAVLT (Rey, 1958), and Mattis Dementia Rating Scale, 2nd Edition (DRS-2; Jurica, Leittten, \& Mattis, 2001). They were excluded if they scored below 25 on the MMSE or 1.5 standard deviations below age-appropriate means on RAVLT delayed recall (Woodard et al., 2009). A score below 10 on the Geriatric Depression Scale (GDS; Yesavage et al., 1983) was also required for inclusion in the study to rule out moderate to severe depressive symptoms, as was a score in the normal range on a measure of activities of daily living (Lawton Instrumental Activities of Daily Living Scale; Lawton \& Brody, 1969). Results of baseline neuropsychological testing is presented in Table 1.

\section{Longitudinal Episodic Memory Testing}

Participants underwent repeat RAVLT testing at the 3-year follow-up examination. The RAVLT was chosen as the primary outcome measure based on findings that EM tests are the most sensitive to early cognitive decline in healthy, non-demented elders (Albert et al., 2001; Backman et al., 2001; Blacker et al., 2007; Bondi et al., 1999). From the RAVLT, three primary indices were investigated: total recall of trials $1-5$, immediate recall, and delayed recall. Participants received alternate forms of the RAVLT, with a counterbalanced order of administration; no significant differences in performance accuracy were observed across the test forms.

\section{MRI Scanning}

At study entry participants underwent anatomical and DTI scans. Images were acquired on a General Electric (Waukesha, WI) Signa Excite 3.0 Tesla short bore scanner at Froedtert Memorial Lutheran Hospital (Milwaukee, WI). Foam padding was used to reduce head movement within the coil. 
NOT THE PUBLISHED VERSION; this is the author's final, peer-reviewed manuscript. The published version may be accessed by following the link in the citation at the bottom of the page.

\section{Diffusion tensor imaging}

Whole brain ( 35 axial slices; $4 \mathrm{~mm}$ ) diffusion tensor images were acquired with 25 encoding directions $\left(b=1000 \mathrm{~s} / \mathrm{mm}^{2}\right.$; echo time $[T E]=\min (86.5 \mathrm{~ms})$; repetition time $[T R]=10,000 \mathrm{~ms}$; field of view $[\mathrm{FOV}]=240 \mathrm{~mm}$, matrix $=128 \times 128$; acquisitions=1). Images were smoothed at $6 \mathrm{~mm}$ full-width half-maximum to eliminate Gibbs ringing. Head movement and eddy currents were corrected and the Brain Extraction Tool (BET; Smith, 2002) was applied to exclude nonbrain voxels. Each diffusion-weighted image was registered to the corresponding B0 image (no diffusion encoding). FA, MD, DA, and DR images were created by fitting a tensor model to the raw diffusion data using the FSL Diffusion Toolbox (Behrens et al., 2003).

DTI data were first processed using the Tract-Based Spatial Statistics (TBSS; Smith et al., 2006) program found in FSL 4.0 image processing software (http://www.fmrib.ox.ac.uk/fsl/tbss/index.html) to create a mean WM skeleton. Specifically, all FA images were aligned to the FMRIB58 standard image in cubic $1 \mathrm{~mm}$ standard space. FA images were averaged and a skeleton created with an FA threshold of 0.2 representing tracts common to all participants. This method of "thinning" each WM tract perpendicular to the tract and thresholding FA helps minimize partial volume effects and areas of high intersubject variability (Smith et al., 2006). Each participant's maximum FA value from the nearest relevant tract center was assigned to the corresponding skeleton voxel, which corrects for residual misalignments after nonlinear registration. Diffusivity measures from the same maximum FA voxels were also assigned to the corresponding skeleton voxels.

Three WM tracts of interest (TOIs) were defined because of their importance for EM: the bilateral cingulate-hippocampal tract (CGH), fornix/stria terminalis (FxS), and uncinate fasciculus (UNC). These TOIs (see Figure 1) were derived from the ICBM DTI-81 WM atlas (Mori et al., 2008). Using the Analysis of Functional Neuroimages (AFNI) software package (Cox, 1996), the TBSS-derived FA skeleton was overlaid onto the three DTI-81-defined TOIs. From this conjunction mask, mean FA, MD, DA, and DR values were calculated for each subject for each TOI. Figure 1 displays these tracts as represented in the ICBM DTI-81 atlas. 

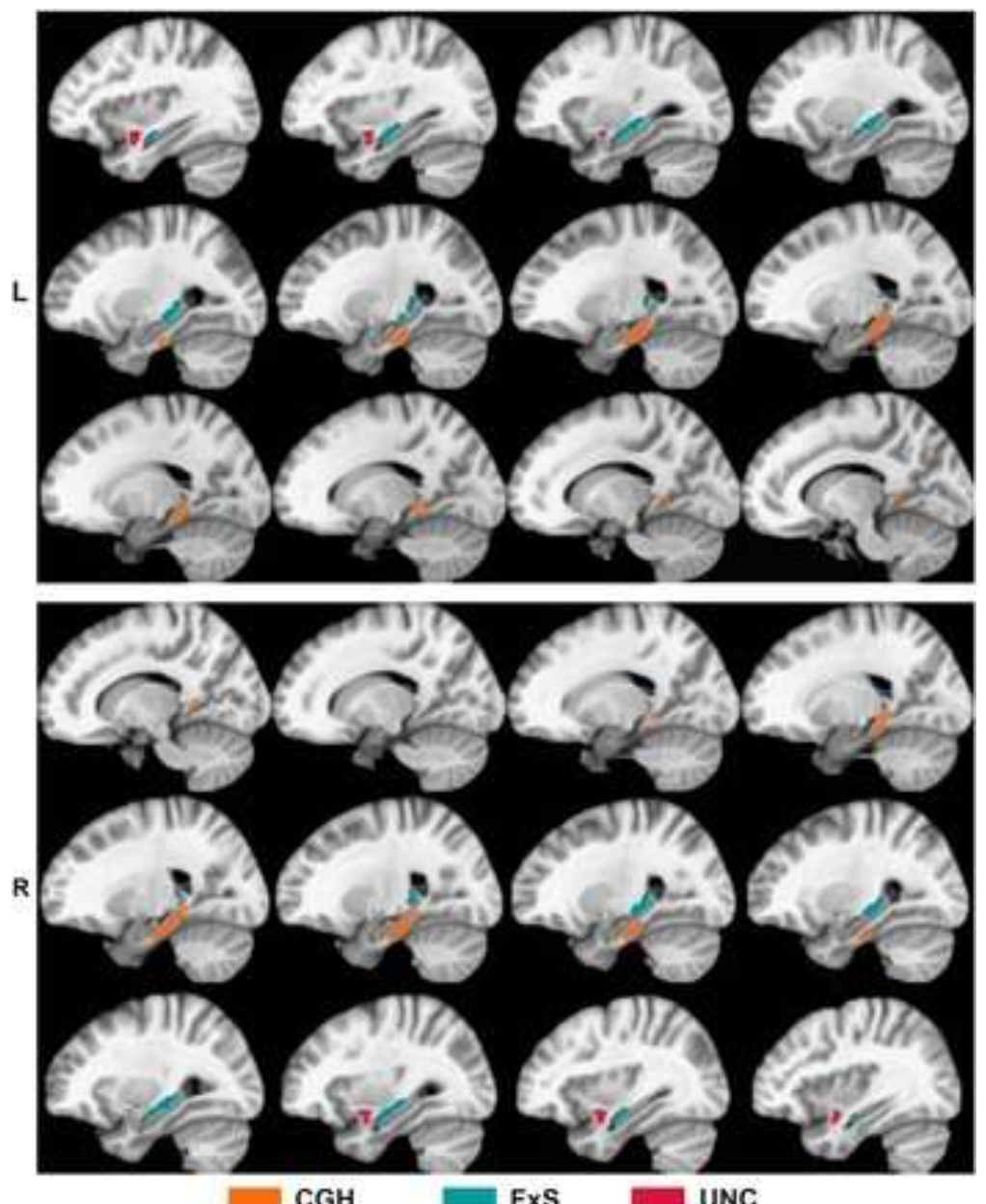

Fig. 1 Three medial temporal white matter tracts investigated in this study: cingulatehippocampal (CGH), fornix/stria-terminalis (FXS), and uncinate fasciculus (UNC). Tracts defined by the ICBM DTI-81 white matter atlas (Mori et al., 2008).

\section{Anatomical MRI}

High-resolution anatomical scans were derived from a threedimensional spoiled gradient-recalled at steady-state (SPGR) pulse sequence $[\mathrm{TE}=3.9 \mathrm{~ms} ; \mathrm{TR}=9.5 \mathrm{~ms}$; inversion recovery preparation time $=450 \mathrm{~ms}$; flip angle $=12$ degrees; number of excitations $=2$; slice thickness $=1.0 \mathrm{~mm}$; $\mathrm{FOV}=24 \mathrm{~cm}$; resolution $=256 \times 224$; slices $=144]$. Bilateral volumes of selected GM (hippocampus, parahippocampal gyrus, entorhinal cortex) and WM (parahippocampal gyrus, entorhinal cortex) regions related to DTI tracts of interest as well as total intracranial volume (ICV) were calculated from SPGR images using FreeSurfer software version 5.1.0 (Fischl et al., 2004). GM and WM 
NOT THE PUBLISHED VERSION; this is the author's final, peer-reviewed manuscript. The published version may be accessed by following the link in the citation at the bottom of the page.

volumes were then corrected for variations in head size by dividing by ICV.

\section{Statistical Analyses}

Prediction of change in EM performance was accomplished by correlating residualized 3-year change score outcomes on three EM measures (RAVLT total recall of trials 1-5, RAVLT immediate recall, and RAVLT delayed recall) with the following set of baseline (study entry) predictors: DTI (FA, MD, DA, DR), volumetric MRI, demographic characteristics, $A D$ genetic risk factors (APOE, FH), and AD metabolic risk factors (hypertension, hypercholesterolemia, and elevated triclycerides). Residualized change scores were calculated by regressing baseline scores on 3-year follow-up scores; this technique adjusts for baseline performance, practice effects and regression to the mean (McSweeny, Naugle, Chelune, \& Luders, 1993; Tucker, Damarin, \& Messick, 1966). Pearson and point biserial correlations were calculated for continuous and dichotomous predictor variables, respectively. To control for multiple comparisons, a false discovery rate (FDR; Benjamini \& Hochberg, 1995) threshold was used to control family-wise error rate with significance established at $\mathrm{q}<.05$. FDR thresholds were assessed separately for each class of DTI (FA, MD, $D A$, and $D R$ ), volumetric (GM and WM), and non-imaging variables. Multiple regression was performed when one or more of the correlations significantly predicted the same RAVLT outcome measure following FDR correction. We also report, but do not interpret, $p<.05$ correlations that did not survive FDR correction.

\section{Results}

Over the 3 -year retest interval, seven participants (14\%) converted to a diagnosis of MCI based on our previously published criteria (see Woodard et al., 2009 for details), five of whom (71\%) were APOE $\varepsilon 4$ carriers. A Fisher's exact test indicated a significantly higher proportion of carriers amongst those who converted to $\mathrm{MCI}$ $(p=.034)$, while no significant association was found between the presence/absence of $\mathrm{FH}$ of dementia and MCI conversion. 
Table 2 summarizes correlations between baseline DTI tract measures and residualized change scores on the three EM measures: RAVLT total recall of trials $1-5$, RAVLT immediate recall, and RAVLT delayed recall. Of the FA tract measures, only 1 of 18 correlations survived FDR correction: right CGH tract correlated negatively with 3year change in RAVLT total recall of trials $1-5(r=-.381 ; p=.006)$.

Table 2. Pearson product-moment correlations between baseline DTI indices for each WM tract and residualized RAVLT change scores

\begin{tabular}{|c|c|c|c|}
\hline & \multicolumn{3}{|c|}{ RAVLT residualized change scores } \\
\hline & $\begin{array}{c}\text { Trials } \\
1-5\end{array}$ & $\begin{array}{l}\text { Immediate } \\
\text { recall }\end{array}$ & $\begin{array}{c}\text { Delayed } \\
\text { recall }\end{array}$ \\
\hline \multicolumn{4}{|c|}{ Fractional anisotropy } \\
\hline Left CGH & -.201 & -.159 & -.181 \\
\hline Right $\mathrm{CGH}$ & -.381 & -331 & -.342 \\
\hline Left FxS & -.119 & -.093 & -.074 \\
\hline Right FxS & -.035 & .183 & .201 \\
\hline Left UNC & -.212 & -165 & -108 \\
\hline Right UNC & -.233 & -.144 & -.147 \\
\hline \multicolumn{4}{|c|}{ Mean diffusivity } \\
\hline Left CGH & -.171 & -.346 & -.500 \\
\hline Right $\mathrm{CGH}$ & -.160 & -.140 & -.316 \\
\hline Left FxS & .054 & 147 & .021 \\
\hline Right FxS & .051 & -.031 & -.168 \\
\hline Left UNC & -.199 & -189 & -.375 \\
\hline Right UNC & -.022 & -.134 & -.308 \\
\hline \multicolumn{4}{|c|}{ Axial diffusivity } \\
\hline Left CGH & -.265 & -.390 & -.561 \\
\hline Right CGH & -.351 & -.289 & -.424 \\
\hline Left FxS & -.050 & .069 & -.068 \\
\hline Right FxS & .034 & .067 & -.085 \\
\hline Left UNC & -.394 & -.315 & -.459 \\
\hline Right UNC & -.109 & -161 & -.346 \\
\hline \multicolumn{4}{|c|}{ Radial diffusivity } \\
\hline Left CGH & -.076 & -.266 & -.386 \\
\hline Right CGH & .056 & .025 & -.103 \\
\hline Left FxS & .106 & .163 & .070 \\
\hline Right FxS & -.026 & -.109 & -.284 \\
\hline Left UNC & -.027 & -.030 & -.188 \\
\hline Right UNC & .171 & .070 & -.068 \\
\hline
\end{tabular}

Bold/italics indicate correlations surviving FDR correction for multiple comparisons. Italics indicate correlations significant at $p<.05$, but not surviving FDR correction.

Journal of the International Neuropsychological Society, Vol 22, No. 10 (November 2016): pg. 1005-1015. DOI. This article is (C) Cambridge University Press and permission has been granted for this version to appear in ePublications@Marquette. Cambridge University Press does not grant permission for this article to be further copied/distributed or hosted elsewhere without the express permission from Cambridge University Press. 
$\mathrm{CGH}=$ cingulate-hippocampal tract; $\mathrm{FxS}=$ fornix/stria terminalis tract; UNC=uncinate fasciculus tract; RAVLT = Rey Auditory Verbal Learning Test.

Significant negative correlations between MD and RAVLT delayed recall were observed bilaterally in the $\mathrm{CGH}$ and UNC tracts (Table 2). These results indicate that higher baseline MD was associated with decreased memory performance over time. Since MD represents an average of DA and DR, we then conducted separate analyses to determine if these significant MD correlations were being driven by DA, DR, or both.

Similar to MD, baseline DA correlated negatively with changes in RAVLT delayed recall change scores in the bilateral CGH and UNC tracts (see Table 2; Figure 2). In addition, DA correlated negatively with RAVLT immediate recall in the left CGH and RAVLT total recall of trials 1-5 in the left UNC (Table 2).
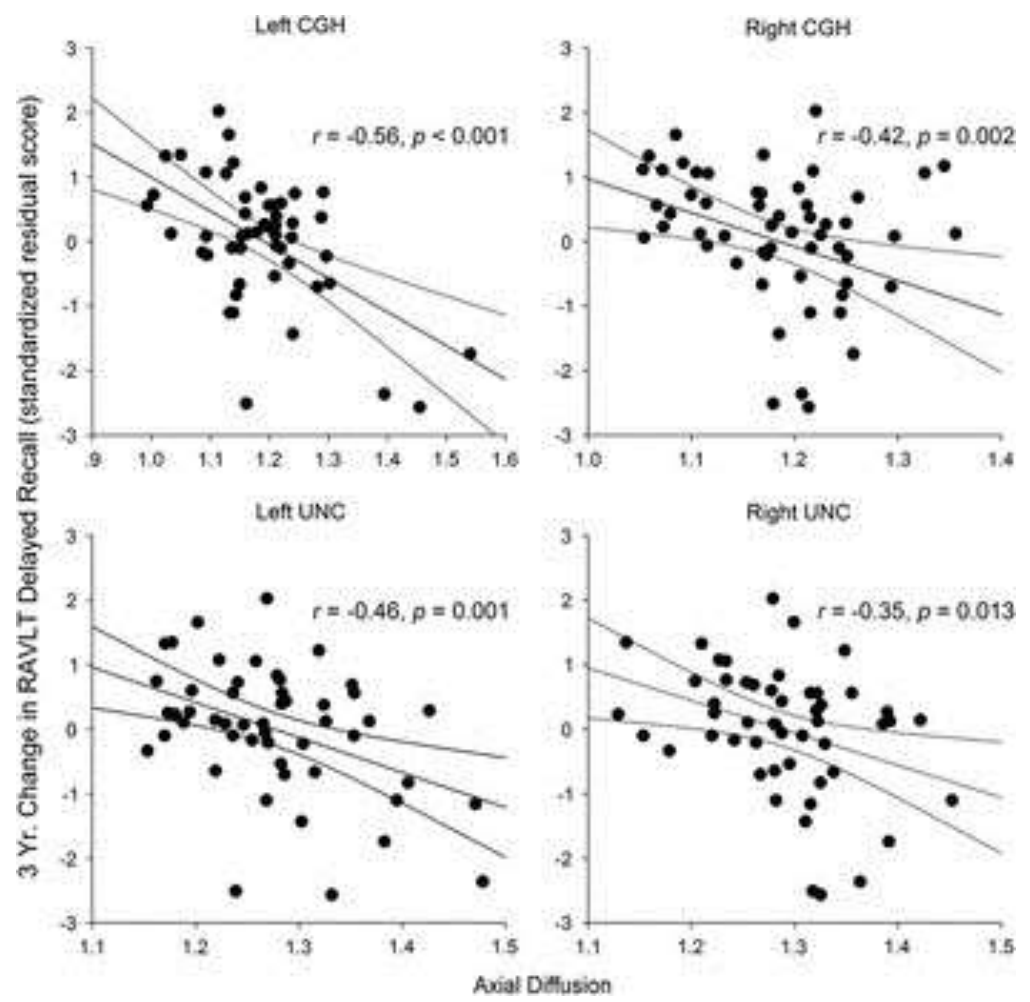

Fig. 2 Correlations between baseline axial diffusion and 3-year changes in Rey Auditory Verbal Learning Test (RAVLT) delayed recall scores. Regression line includes the $95 \%$ confidence interval. $\mathrm{CGH}=$ cingulate-hippocampal tract; UNC=uncinate fasciculus. 
NOT THE PUBLISHED VERSION; this is the author's final, peer-reviewed manuscript. The published version may be accessed by following the link in the citation at the bottom of the page.

The relationship between baseline DR and changes in RAVLT test performance was less pronounced. Only 1 of 18 correlations was significant: the left $\mathrm{CGH}$ tract correlated negatively with changes in RAVLT delayed recall (Table 2). These results indicate that the significant correlations between MD and RAVLT delayed recall scores were being driven primarily by baseline axial rather than radial diffusion.

Table 3 summarizes correlations between baseline MTL GM and WM volumes and changes in RAVLT test performance. None of these correlations were significant after FDR correction. These results indicate that DTI measures of WM microstructure are better at predicting changes in EM test performance than measures of atrophy within MTL structures.

Table 3 Pearson product-moment correlations between baseline mesial temporal lobe gray and white matter volumes and residualized RAVLT change scores

\begin{tabular}{|c|c|c|c|}
\hline & \multicolumn{3}{|c|}{ RAVLT residualized change scores } \\
\hline & $\begin{array}{c}\text { Trials } \\
1-5\end{array}$ & $\begin{array}{l}\text { Immediate } \\
\text { recall }\end{array}$ & $\begin{array}{c}\text { Delayed } \\
\text { recaill }\end{array}$ \\
\hline \multicolumn{4}{|l|}{ Gray matter Folume } \\
\hline L Hippocampus & 088 & .026 & -.065 \\
\hline R Hippocamplus & .145 & 171 & 097 \\
\hline L Entorhinal cortex & -.116 & $\approx_{4} 105$ & -.032 \\
\hline R Entorhinal cortex & 247 & .284 & .156 \\
\hline L Parahippocampal gyrus & 015 & 016 & $= \pm 029$ \\
\hline R Parahippocampal gyrus & $=037$ & .024 & -.039 \\
\hline \multicolumn{4}{|l|}{ White matter yolume } \\
\hline L Entorhinal cortex & -220 & -.255 & -.176 \\
\hline R Entorhinal cortex & .047 & .066 & -.003 \\
\hline L Parahippocampal gyrus & .102 & 065 & .039 \\
\hline R Parahippocampal gyrus & -.033 & -.033 & $=.096$ \\
\hline
\end{tabular}

Bold/italics indicate correlations surviving FDR correction for multiple comparisons. Italics indicate correlations significant at $\mathrm{p}<.05$, but not surviving FDR correction. RAVLT=Rey Auditory Verbal Learning Test.

Table 4 reports correlations between 3-year change in RAVLT scores and demographic characteristics, genetic risk factors, and metabolic risk factors at baseline. Only one correlation was significant: 
change in RAVLT immediate recall correlated negatively with APOE status (i.e., carriers exhibited poorer performance over time).

Table 4 Correlations between demographic, AD genetic risk, and metabolic risk variables and residualized RAVLT change scores

\begin{tabular}{|c|c|c|c|}
\hline & \multicolumn{3}{|c|}{ RAVLT Residualized Change Scores } \\
\hline & $\begin{array}{c}\text { Trials } \\
1-5\end{array}$ & $\begin{array}{l}\text { Immediate } \\
\text { recall }\end{array}$ & $\begin{array}{c}\text { Delayed } \\
\text { recall }\end{array}$ \\
\hline \multicolumn{4}{|l|}{ Demographics } \\
\hline Age & 089 & .005 & -184 \\
\hline Education & -.206 & -1006 & -015 \\
\hline $\operatorname{Sex} 0$ & -029 & -0.075 & -.022 \\
\hline \multicolumn{4}{|l|}{$\mathrm{AD}$ risk factors } \\
\hline APOE 4400 & -.257 & -.385 & -263 \\
\hline Family historyou & -.131 & -190 & -156 \\
\hline \multicolumn{4}{|l|}{ Metabolic risk variables } \\
\hline Hypertension & .024 & .150 & -116 \\
\hline High triglycerides:0 & -.285 & -146 & -.233 \\
\hline Hypercholesterolemiat: & -115 & -.154 & -148 \\
\hline
\end{tabular}

*Pearson corelations; **Point biserial correlations.

Bold/italics indicate correlations surviving FDR correction for multiple comparisons. Italics indicate correlations significant at $p<.05$, but not surviving FDR correction. RAVLT=Rey Auditory Verbal Learning Test.

Because both DA within the left CGH tract (Table 2) and APOE status (Table 4) correlated significantly with changes in RAVLT immediate recall, a multiple regression was performed with RAVLT immediate recall as the outcome and left CGH DA and APOE status as predictors. Both APOE status $(\beta=-.334 ; t(50)=-2.609 ; p=.012)$ and left CGH DA $(\beta=-.327 ; t(50)=-2.556, p=.014)$ remained significant predictors in the overall model $\left(F(2,48)=8,242 ; p=.001 ; \mathrm{R}^{2}=.260\right)$, suggesting that each variable made independent contributions to the prediction of longitudinal EM changes.

\section{Discussion}

Our results indicate that DTI diffusion measures (predominantly DA and MD) of mesial temporal lobe white matter tracts (cingulatehippocampal and uncinate fasciculus) obtained from cognitively intact older adults predicted subsequent episodic memory decline over a 3year interval. It is important to emphasize that these results were 
driven primarily by DA, since MD represents an average of DR and DA measures. Specifically, greater DA in these two critical EM-related WM tracts predicted a 3-year decline predominantly in delayed free recall on the RAVLT (Table 2, Figure 2). FA and DR in the cingulatehippocampal tract also predicted EM change, although these results were less striking. Left hemisphere structures were more predictive of verbal EM decline overall, but most of the significant correlations between DTI measures and EM change involved bilateral WM tracts. Both APOE \&4 status and DA within the left CGH tract made unique and independent contributions to the prediction of change in RAVLT immediate recall. In contrast, volumes of MTL WM and GM structures did not predict 3-year EM change (Table 3). Likewise, demographic variables (age, education, and sex), $\mathrm{FH}$ of dementia, and metabolic/vascular risk factors (hypertension, hypercholesterolemia, and elevated triglycerides) did not predict long-term changes in EM functions (Table 4).

It is important to note that, in the current study, microstructural changes in WM, as reflected predominantly by increased DA within the CGH and UNC tracts, were able to predict EM changes, whereas atrophy of the MTL GM and WM structures was not. Abnormalities in DA are commonly associated with axonal disruption (Agosta et al., 2011; O'Dwyer et al., 2011; Song et al., 2003) that likely precede neuronal loss and atrophy as observed on anatomical MR scans, whereas DR has been associated with demyelination or myelin breakdown (Song et al., 2002). Recent studies, however, suggest this explanation may be overly simplistic (Pierpaoli et al., 2001; WheelerKingshott \& Cercignani, 2009). Consistent with our findings, DA was found to be more sensitive than DR in studies of cognitive decline on a general cognitive screening measure in healthy older adults (Wang et al., 2015) and in conversion to MCI and AD in healthy elders (Fletcher et al., 2013). DA also appears more sensitive than other DTI measures to WM changes along the symptomatic stage of the AD neurodegenerative continuum (Acosta-Cabronero et al., 2010; Agosta et al., 2011). Thus DA changes may serve as an earlier and more sensitive biomarker than atrophy for predicting future EM decline, especially in cognitively intact, healthy elders.

DTI abnormalities in the CGH and UNC tracts have been observed in prior studies involving healthy elders and patients with

Journal of the International Neuropsychological Society, Vol 22, No. 10 (November 2016): pg. 1005-1015. DOI. This article is (c) Cambridge University Press and permission has been granted for this version to appear in e-

Publications@Marquette. Cambridge University Press does not grant permission for this article to be further copied/distributed or hosted elsewhere without the express permission from Cambridge University Press. 
MCI and AD. The CGH tract consists of posterior cingulate projections to the parahippocampal gyrus, the major input system to the hippocampus (Dickerson \& Eichenbaum, 2010). DTI measures from this tract have previously been shown to predict EM decline (Zhuang et al., 2012) and general cognitive decline (Wang et al., 2015) in healthy elders. The UNC tract, which provides an important connection between MTL structures including the hippocampus, amygdala, and orbitofrontal cortex (Von Der Heide, Skipper, Klobusicky, \& Olson, 2013), has a demonstrated role in verbal EM performance (Christidi et al., 2014; Schaeffer et al., 2014). Diffusivity measures (DA and DR) in both the CGH and UNC tracts are altered in MCI and AD (Canu et al., 2010; Fu, Liu, Li, Chang, \& Li, 2014; Hiyoshi-Taniguchi et al., 2015; Salat et al., 2010; Yasmin et al., 2008; Zhang et al., 2007), and have shown negative associations with EM in MCI (Li et al., 2014; MetzlerBaddeley et al., 2012; Remy, Vayssiere, Saint-Aubert, Barbeau, \& Pariente, 2015; Zhuang et al., 2013).

Surprisingly, DTI measures derived from the fornix, the major WM output tract from the hippocampus, did not predict 3-year changes in EM. Metzler-Baddeley et al. (2012) observed that whereas DTI measures correlated with EM performance in healthy older adults, DTI measures from the uncinate and parahippocampal tracts, but not the fornix, correlated with EM in MCI patients. They hypothesized that early fornix damage associated with the AD pathology may cause other associated WM tracts to become disproportionately involved in EM function. The finding by Ray et al. (2015) that MCI participants' performance on EM measures was more related to parahippocampal cortex than fornix volume also supports this hypothesis.

The current findings suggest that DTI of MTL tracts may provide important information for the detection of preclinical changes associated with AD (Sperling et al., 2011). Our sample was enriched with individuals at increased genetic risk of $A D$ by virtue of possessing at least one APOE \&4 allele and/or having FH of dementia. We focused our DTI analysis on fiber tracts within the MTL, since this region is affected early in the AD process and is less susceptible to the effects of normal aging (Raz \& Rodrigue, 2006). In addition, as hypothesized, DTI measures were observed to be most effective in predicting changes in delayed recall, one of the earliest cognitive indicators of conversion to AD in asymptomatic elders (Albert et al., 2001; Hamel et 
al., 2015; Mistridis et al., 2015; Papp et al., 2015). We also note that a significantly higher proportion of our APOE $\varepsilon 4$ carriers than noncarriers converted to MCI at the 3-year follow-up examination, suggesting that our recruitment procedure likely resulted in a higher proportion of persons in the preclinical stage of $A D$ than the general population of similarly-aged adults. Post hoc supplementary moderated regression analyses were conducted to examine the interaction effects of WM microstructure and APOE $\varepsilon 4$ status on EM change. These analyses were not significant, suggesting that APOE $\varepsilon 4$ and early WM changes may be independent contributors to EM decline.

It is important to note that we did not directly measure amyloid $\beta$ and tau derived from cerebrospinal fluid (CSF) nor did we conduct amyloid positron emission tomography (PET) imaging. Such measures are also able to predict subsequent cognitive decline in healthy elders (Ellis et al., 2013; Fagan et al., 2007; Lim et al., 2013; Mattsson et al., 2015; Moghekar et al., 2013; Thai et al., 2015). It should also be noted, however, that not all healthy adults with evidence of amyloid $\beta$ or abnormal tau demonstrate cognitive decline (Edmonds, DelanoWood, Galasko, Salmon, \& Bondi, 2015; Sperling et al., 2011). A recent study of cognitively normal elders from the ADNI database (Edmonds et al., 2015) demonstrated that 17-22\% of participants with evidence of cerebral amyloid accumulation on PET imaging converted to MCI or AD over a period of 12-96 months. Adding abnormal CSF tau to the presence of amyloidosis increased the prediction to $37 \%$. These findings suggest that additional neuroimaging markers may be needed to improve the prediction of conversion to MCI and/or AD in healthy older adults. Future larger scale longitudinal studies are needed to compare the sensitivity of DTI measures to other imaging techniques (anatomical MRI, amyloid and tau PET) and CSF biomarkers in predicting EM decline and conversion from the cognitively healthy state to MCI and AD.

The current study is not without limitations. Despite enriching our sample with persons with a higher genetic risk for developing $A D$, only seven participants converted to MCI after the three year follow-up interval. This sample size is too small to draw conclusions about the accuracy of DTI to predict AD progression. Our tracts-of-interest were based on a WM skeleton created in TBSS using the ICBN DTI-81 atlas (Mori et al., 2008). By focusing on the WM skeleton based on the 
highest FA values, we reduced, but possibly did not entirely eliminate, partial volume effects. Finally, metabolic/vascular risk factors were based on self-report.

To summarize, our findings suggest that DTI measures of axial diffusion within MTL WM tracts are capable of predicting EM decline in healthy, cognitively intact older adults. Such information may also help in characterizing the pathophysiological changes associated with the preclinical stage of AD. With data from future larger scale longitudinal validation studies, it is conceivable that DTI measures will prove useful, in combination with other imaging and non-imaging biomarkers, in the identification of healthy individuals at greatest risk for cognitive decline to serve in AD prevention studies and for monitoring the effects of interventions administered during the preclinical stage.

\section{Acknowledgments}

We thank Alissa M. Butts, Kelli L. Douville, Christina M. Figueroa, Amelia Gander, Evan Gross, Leslie M. Guidotti-Breting, Nathan C. Hantke, Kathleen E. Hazlett, Emily Hoida, Cassandra Kandah, Christina D. Kay, Dana Kelly, Monica Matthews, Sarah K. Miller, Andria L. Norman, Katherine Reiter, Michael A. Sugarman, and Qi Zhang for their assistance. This work was supported by the National Institutes of Health Grants R01 AG022304 and M01 RR00058. The content is solely the responsibility of the authors and does not necessarily represent the official views of the National Institute on Aging or the National Institutes of Health. Conflict of interest: The authors declare no conflict of interest.

\section{References}

J. Acosta-Cabronero , G.B. Williams , G. Pengas , \& P.J. Nestor (2010). Absolute diffusivities define the landscape of white matter degeneration in Alzheimer's disease. Brain, 133, 529-539.

F. Agosta , M. Pievani , S. Sala , C. Geroldi , S. Galluzzi , G.B. Frisoni , \& M. Filippi (2011). White matter damage in Alzheimer disease and its relationship to gray matter atrophy. Radiology, 258(3), 853-863. doi: 10.1148/radiol.10101284

M.S. Albert , M.B. Moss , R. Tanzi , \& K. Jones (2001). Preclinical prediction of AD using neuropsychological tests. Journal of the International Neuropsychological Society, 7(5), 631-639. 
G.S. Alves, L. O’Dwyer , A. Jurcoane, V. Oertel-Knochel , C. Knochel , D. Prvulovic, \& J. Laks (2012). Different patterns of white matter degeneration using multiple diffusion indices and volumetric data in mild cognitive impairment and Alzheimer patients. PLoS One, 7(12), e52859. doi: 10.1371/journal.pone.0052859

L. Backman , B.J. Small , \& L. Fratiglioni (2001). Stability of the preclinical episodic memory deficit in Alzheimer's disease. Brain, 124(Pt 1), 96102.

M.L. Balthazar , C.L. Yasuda , F. Cendes , \& B.P. Damasceno (2010). Learning, retrieval, and recognition are compromised in aMCI and mild AD: Are distinct episodic memory processes mediated by the same anatomical structures? Journal of the International Neuropsychological Society, 16(1), 205-209. doi: 10.1017/s1355617709990956

G. Bartzokis, D. Sultzer , P.H. Lu , K.H. Nuechterlein , J. Mintz , \& J.L. Cummings (2004). Heterogeneous age-related breakdown of white matter structural integrity: Implications for cortical "disconnection" in aging and Alzheimer's disease. Neurobiology of Aging, 25, 843-851.

M.W. Behrens, M. Woolrich, H. Jenkinson, R.G. Johansen-Berg , S. Nunes, \& P.M. Clare (2003). Characterization and propagation of uncertainty in diffusion-weighted MR imaging. Magnetic Resonance in Medicine, 50, 1077-1088.

Y. Benjamini , \& Y. Hochberg (1995). Controlling the false discovery rate: A practical and powerful approach to multiple testing. Journal of the Royal Statistical Society: Series B (Statistical Methodology), 57, 289300.

I.J. Bennett, \& D.J. Madden (2014). Disconnected aging: Cerebral white matter integrity and age-related differences in cognition.

Neuroscience, 276, 187-205. doi: 10.1016/j.neuroscience.2013.11.026

D. Blacker , H. Lee , A. Muzikansky , E.C. Martin , R. Tanzi , J.J. McArdle , \& M. Albert (2007). Neuropsychological measures in normal individuals that predict subsequent cognitive decline. Archives of Neurology, 64(6), 862-871. doi: 10.1001/archneur.64.6.862

M.W. Bondi , D.P. Salmon, D. Galasko, R.G. Thomas, \& L.J. Thal (1999). Neuropsychological function and apolipoprotein $\mathrm{E}$ genotype in the preclinical detection of Alzheimer's disease. Psychology and Aging, 14(2), 295-303.

E. Canu , D.G. McLaren , M.E. Fitzgerald , B.B. Bendlin , G. Zoccatelli , F. Alessandrini , \& G.B. Frisoni (2010). Microstructural diffusion changes are independent of macrostructural volume loss in moderate to severe Alzheimer's disease. Journal of Alzheimer's Disease, 19(3), 963-976. doi: $10.3233 /$ jad-2010-1295 
NOT THE PUBLISHED VERSION; this is the author's final, peer-reviewed manuscript. The published version may be

accessed by following the link in the citation at the bottom of the page.

V.A. Cardenas, L.L. Chao, C. Studholme, K. Yaffe, B.L. Miller , C. Madison, \& M.W. Weiner (2011). Brain atrophy associated with baseline and longitudinal measures of cognition. Neurobiology of Aging, 32(4), 572580. doi: 10.1016/j.neurobiolaging.2009.04.011

R.J. Caselli , E.M. Reiman , D. Osborne, J.G. Hentz , L.C. Baxter , J.L. Hernandez , \& G.G. Alexander (2004). Longitudinal changes in cognition and behavior in asymptomatic carriers of the APOE e4 allele. Neurology, 62(11), 1990-1995.

F. Christidi , I. Zalonis, S. Kyriazi , M. Rentzos , E. Karavasilis , E.A. Wilde , \& I. Evdokimidis (2014). Uncinate fasciculus microstructure and verbal episodic memory in amyotrophic lateral sclerosis: A diffusion tensor imaging and neuropsychological study. Brain Imaging and Behavior, 8(4), 497-505. doi: 10.1007/s11682-013-9271-y

R. Cox (1996). AFNI: Sotware for analysis and visualization of functional magnetic resonance images. Computers and Biomedical Research, 29, 162-173.

T. den Heijer, M.I. Geerlings, F.E. Hoebeek, A. Hofman , P.J. Koudstaal , \& M.M. Breteler (2006). Use of hippocampal and amygdalar volumes on magnetic resonance imaging to predict dementia in cognitively intact elderly people. Archives of General Psychiatry, 63(1), 57-62. doi: 10.1001/archpsyc.63.1.57

B.C. Dickerson , \& H. Eichenbaum (2010). The episodic memory system: Neurocircuitry and disorders. Neuropsychopharmacology, 35(1), 86104. doi: $10.1038 / n p p .2009 .126$

E.C. Edmonds, L. Delano-Wood, D.R. Galasko, D.P. Salmon , \& M.W. Bondi (2015). Subtle cognitive decline and biomarker staging in preclinical Alzheimer's disease. Journal of Alzheimer's Disease, 47(1), 231-242. doi: $10.3233 /$ jad-150128

K.A. Ellis , Y.Y. Lim , K. Harrington , D. Ames , A.I. Bush , D. Darby , ... AIBL Research Group. (2013). Decline in cognitive function over 18 months in healthy older adults with high amyloid-beta. Journal of Alzheimer's Disease, 34(4), 861-871. doi: 10.3233/jad-122170

A. Ezzati , M.J. Katz , M.L. Lipton, M.E. Zimmerman , \& R.B. Lipton (2016). Hippocampal volume and cingulum bundle fractional anisotropy are independently associated with verbal memory in older adults. Brain Imaging and Behavior, 10, 652-659. doi: 10.1007/s11682-015-9452y

A.M. Fagan , C.M. Roe , C. Xiong , M.A. Mintun, J.C. Morris , \& D.M. Holtzman (2007). Cerebrospinal fluid tau/beta-amyloid(42) ratio as a prediction of cognitive decline in nondemented older adults. Archives of Neurology, 64(3), 343-349. doi: 10.1001/archneur.64.3.noc60123 
B. Fischl , D.H. Salat , E. Busa, M. Albert , M. Dieterich , \& C. Haselgrove (2004). Whole brain segmentation: Automated labeling of neuroanatomical structures in the human brain. Neuron, 33, 341-355.

E. Fletcher , M. Raman , P. Huebner , A. Liu , D. Mungas , O. Carmichael , \& C. DeCarli (2013). Loss of fornix white matter volume as a predictor of cognitive impairment in cognitively normal elderly individuals. JAMA Neurology, 70(11), 1389-1395. doi: 10.1001/jamaneurol.2013.3263

M.F. Folstein , S.E. Folstein , \& P.R. McHugh (1975). "Mini-Mental State": A practical method for grading the cognitive state of patients for the clinician. Journal of Psychiatry Research, 12, 189-198.

J.L. Fu , Y. Liu , Y.M. Li , C. Chang , \& W.B. Li (2014). Use of diffusion tensor imaging for evaluating changes in the microstructural integrity of white matter over 3 years in patients with amnesic-type mild cognitive impairment converting to Alzheimer's disease. Journal of Neuroimaging, 24(4), 343-348. doi: 10.1111/jon.12061

N. Geschwind (1965a). Disconnexion syndromes in animals and man. I. Brain, 88(2), 237-294.

N. Geschwind (1965b). Disconnexion syndromes in animals and man. II. Brain, 88(3), 585-644.

J. Golomb , A. Kluger, M.J. de Leon , S.H. Ferris, M. Mittelman , J. Cohen , \& A.E. George (1996). Hippocampal formation size predicts declining memory performance in normal aging. Neurology, 47(3), 810-813.

R. Hamel , S. Kohler, N. Sistermans , T. Koene, Y. Pijnenburg , W. van der Flier , \& I. Ramakers (2015). The trajectory of cognitive decline in the pre-dementia phase in memory clinic visitors: Findings from the 4CMCI study. Psychological Medicine, 45(7), 1509-1519. doi: $10.1017 / \mathrm{s} 0033291714002645$

K. Hiyoshi-Taniguchi , N. Oishi , C. Namiki , J. Miyata , T. Murai , A. Cichocki , \& H. Fukuyama (2015). The uncinate fasciculus as a predictor of conversion from aMCI to Alzheimer disease. Journal of Neuroimaging, 25, 748-753. doi: $10.1111 /$ jon.12196

P.J. Jurica, C.L. Leittten , \& S. Mattis (2001). Dementia Rating Scale-2 professional manual. Lutz, FL: Psychological Assessment Resources.

M.P. Lawton , \& E.M. Brody (1969). Assessment of older people: Selfmaintaining instrumental activities of daily living. Gerontologist, 9, 179-186.

W. Li , L.T. Muftuler, G. Chen, B.D. Ward, M.D. Budde, J.L. Jones, \& J.S. Goveas (2014). Effects of the coexistence of late-life depression and mild cognitive impairment on white matter microstructure. Journal of the Neurological Sciences, 338(1-2), 46-56. doi:

10.1016/j.jns.2013.12.016

Y.Y. Lim , R.H. Pietrzak , K.A. Ellis, J. Jaeger, K. Harrington , T. Ashwood, \& P. Maruff (2013). Rapid decline in episodic memory in healthy older 
adults with high amyloid-beta. Journal of Alzheimer's Disease, 33(3), 675-679. doi: 10.3233/jad-2012-121516

D.A. Loewenstein , W.W. Barker, J.Y. Chang , A. Apicella , F. Yoshii , P. Kothari , \& R. Duara (1989). Predominant left hemisphere metabolic dysfunction in dementia. Archives of Neurology, 46(2), 146-152.

P. Mattsson , A. Forsberg , J. Persson , L. Nyberg , L.G. Nilsson , C. Halldin , \& L. Farde (2015). beta-Amyloid binding in elderly subjects with declining or stable episodic memory function measured with PET and [(1)(1)C]AZD2184. European Journal of Nuclear Medicine and Molecular Imaging, 42(10), 1507-1511. doi: 10.1007/s00259-0153103-9

A.J. McSweeny, R.I. Naugle, G.J. Chelune, \& H. Luders (1993). "T scores for change": An illustration of a regression approach to depicting change in clinical neuropsychology. The Clinical Neuropsychologist, 7, 300312.

M.M. Mesulam (1990). Large-scale neurocognitive networks and distributed processing for attention, language, and memory. Annals of Neurology, 28(5), 597-613. doi: 10.1002/ana.410280502

C. Metzler-Baddeley, S. Hunt , D.K. Jones , A. Leemans , J.P. Aggleton, \& M.J. O'Sullivan (2012). Temporal association tracts and the breakdown of episodic memory in mild cognitive impairment. Neurology, 79(23), 2233-2240. doi: 10.1212/WNL.0b013e31827689e8

P. Mistridis , S. Krumm , A.U. Monsch , M. Berres , \& K.I. Taylor (2015). The 12 years preceding mild cognitive impairment due to Alzheimer's disease: The temporal emergence of cognitive decline. Journal of Alzheimer's Disease, 48(4), 1095-1107. doi: 10.3233/jad-150137

A. Moghekar , S. Li , Y. Lu , M. Li , M.C. Wang , \& M. Albert , ... Biocard Research Team. (2013). CSF biomarker changes precede symptom onset of mild cognitive impairment. Neurology, 81(20), 1753-1758. doi: 10.1212/01.wnl.0000435558.98447.17

S. Mori , K. Oishi , H. Jiang, L. Jiang , X. Li , K. Akhter , \& J. Mazziotta (2008). Stereotaxic white matter atlas based on diffusion tensor imaging in an ICBM template. Neuroimage, 40(2), 570-582. doi: 10.1016/j.neuroimage.2007.12.035

T.M. Nir, N. Jahanshad, J.E. Villalon-Reina , A.W. Toga , C.R. Jack, \& M.W. Weiner , ... Alzheimer's Disease Neuroimaging Initiative. (2013). Effectiveness of regional DTI measures in distinguishing Alzheimer's disease, MCI, and normal aging. Neuroimage: Clinical, 3, 180-195. doi: $10.1016 / j$.nicl.2013.07.006

L. O’Dwyer , F. Lamberton , A.L. Bokde, M. Ewers , Y.O. Faluyi , C. Tanner, \& H. Hampel (2011). Multiple indices of diffusion identifies white matter damage in mild cognitive impairment and Alzheimer's disease. PLoS One, 6(6), e21745. doi: 10.1371/journal.pone.0021745 
K. Oishi , A. Faria , P.C.M. van Zijl , \& S. Mori (2011). MRI atlas of human white matter, (2nd ed.). London: Elsevier.

K.V. Papp , R.E. Amariglio , E.C. Mormino , T. Hedden, M. Dekhytar , K.A. Johnson, \& D.M. Rentz (2015). Free and cued memory in relation to biomarker-defined abnormalities in clinically normal older adults and those at risk for Alzheimer's disease. Neuropsychologia, 73, 169-175. doi: 10.1016/j.neuropsychologia.2015.04.034

C. Pierpaoli , A. Barnett , S. Pajevic, R. Chen , L.R. Penix , A. Virta , \& P. Basser (2001). Water diffusion changes in Wallerian degeneration and their dependence on white matter architecture. Neuroimage, 13(6 Pt 1), 1174-1185. doi: 10.1006/nimg.2001.0765

N.J. Ray, C. Metzler-Baddeley, M.R. Khondoker, M.J. Grothe, S. Teipel , P. Wright, \& M.J. O'Sullivan (2015). Cholinergic basal forebrain structure influences the reconfiguration of white matter connections to support residual memory in mild cognitive impairment. Journal of Neuroscience, 35(2), 739-747. doi: 10.1523/jneurosci.3617-14.2015

N. Raz , \& K.M. Rodrigue (2006). Differential aging of the brain: Patterns, cognitive correlates and modifiers. Neuroscience and Biobehavioral Reviews, 30(6), 730-748. doi: 10.1016/j.neubiorev.2006.07.001

F. Remy, N. Vayssiere, L. Saint-Aubert, E. Barbeau , \& J. Pariente (2015). White matter disruption at the prodromal stage of Alzheimer's disease: Relationships with hippocampal atrophy and episodic memory performance. Neuroimage: Clinical, 7, 482-492. doi: 10.1016/j.nicl.2015.01.014

A. Rey (1958). L'examen clinique en psychologie. Paris: Presses Universitaires de France.

A.C. Rosen , M.W. Prull , J.D. Gabrieli , T. Stoub , R. O'Hara , L. Friedman , \& L. deToledo-Morrell (2003). Differential associations between entorhinal and hippocampal volumes and memory performance in older adults. Behavioral Neuroscience, 117(6), 1150-1160. doi: 10.1037/0735-7044.117.6.1150

D.H. Salat, D.S. Tuch , A.J. van der Kouwe, D.N. Greve, V. Pappu, \& S.Y. Lee (2010). White matter pathology isolates the hippocampal formation in Alzheimer's disease. Neurobiology of Aging, 31, 244-256.

A.M. Saunders , O. Hulette, K.A. Welsh-Bohmer, D.E. Schmechel , B. Crain , \& J.R. Burke (1996). Specificity, sensitivity, and predictive value of apolipoprotein-E genotyping for sporadic Alzheimer's disease. Lancet, 348, 90-93.

D.J. Schaeffer, C.E. Krafft , N.F. Schwarz , L. Chi , A.L. Rodrigue, J.E. Pierce, \& J.E. McDowell (2014). The relationship between uncinate fasciculus white matter integrity and verbal memory proficiency in children. Neuroreport, 25(12), 921-925. doi: 10.1097/wnr.0000000000000204 
NOT THE PUBLISHED VERSION; this is the author's final, peer-reviewed manuscript. The published version may be

accessed by following the link in the citation at the bottom of the page.

M. Seidenberg , L. Guidotti , K.A. Nielson , J.L. Woodard, S. Durgerian , P. Antuono, \& S.M. Rao (2009). Semantic memory activation in individuals at risk for developing Alzheimer disease. Neurology, 73(8), 612-620. doi: 10.1212/WNL.0b013e3181b389ad

S.M. Smith (2002). Fast robust automated brain extraction. Human Brain Mapping, 17, 143-155.

S.M. Smith , M. Jenkinson , H. Johansen-Berg , D. Ruckert , T.E. Nichols , \& C.E. Mackay (2006). Tract-based spatial statistics: Voxelwise analysis of multi-subject diffusion data. Neuroimage, 31, 1487-1505.

S.K. Song , S.W. Sun , W.K. Ju , S.J. Lin , A.H. Cross , \& A.H. Neufeld (2003). Diffusion tensor imaging detects and differentiates axon and myelin degeneration in mouse optic nerve after retinal ischemia. Neuroimage, 20, 1714-1722.

S.K. Song , S.W. Sun , M.J. Ramsbottom , C. Chang , J. Russell , \& A.H. Cross (2002). Dysmyelination revealed through MRI as increased radial (but unchanged axial) diffusion of water. Neuroimage, 17(3), 1429-1436.

R.A. Sperling , P.S. Aisen, L.A. Beckett, D.A. Bennett , S. Craft , A.M. Fagan , \& C.H. Phelps (2011). Toward defining the preclinical stages of Alzheimer's disease: Recommendations from the National Institute on Aging-Alzheimer's Association workgroups on diagnostic guidelines for Alzheimer's disease. Alzheimers and Dementia, 7(3), 280-292. doi: 10.1016/j.jalz.2011.03.003

C. Thai , Y.Y. Lim , V.L. Villemagne , S.M. Laws , D. Ames , \& K.A. Ellis , ... Australian Imaging, Biomarkers and Lifestyle (AIBL) Research Group. (2015). Amyloid-related memory decline in preclinical Alzheimer's disease is dependent on APOE epsilon4 and is detectable over 18months. PLoS One, 10(10), e0139082. doi:

$10.1371 /$ journal.pone.0139082

P.M. Thompson , K.M. Hayashi , G. de Zubicaray , A.L. Janke , S.E. Rose , J. Semple, \& A.W. Toga (2003). Dynamics of gray matter loss in Alzheimer's disease. Journal of Neuroscience, 23(3), 994-1005.

L.R. Tucker , F. Damarin , \& S. Messick (1966). A base-free measure of change. Psychometrika, 31(4), 457-473.

R.J. Von Der Heide, L.M. Skipper, E. Klobusicky, \& I.R. Olson (2013). Dissecting the uncinate fasciculus: Disorders, controversies and a hypothesis. Brain, 136(6), 1692-1707. doi: 10.1093/brain/awt094

M. Vyhnalek , T. Nikolai , R. Andel , Z. Nedelska, E. Rubinova , H. Markova , \& J. Hort (2014). Neuropsychological correlates of hippocampal atrophy in memory testing in nondemented older adults. Journal of Alzheimer's Disease, 42(Suppl 3), S81-S90. doi: 10.3233/jad-132642 R. Wang , L. Fratiglioni , E.J. Laukka, M. Lovden , G. Kalpouzos, L. Keller , \& C. Qiu (2015). Effects of vascular risk factors and APOE epsilon4 on

Journal of the International Neuropsychological Society, Vol 22, No. 10 (November 2016): pg. 1005-1015. DOI. This article is (C) Cambridge University Press and permission has been granted for this version to appear in ePublications@Marquette. Cambridge University Press does not grant permission for this article to be further copied/distributed or hosted elsewhere without the express permission from Cambridge University Press. 
NOT THE PUBLISHED VERSION; this is the author's final, peer-reviewed manuscript. The published version may be accessed by following the link in the citation at the bottom of the page.

white matter integrity and cognitive decline. Neurology, 84(11), 11281135. doi: 10.1212/wnl.0000000000001379

C.A. Wheeler-Kingshott , \& M. Cercignani (2009). About "axial" and "radial" diffusivities. Magnetic Resonance in Medicine, 61(5), 1255-1260. doi: 10.1002/mrm.21965

L.E. Wisse, Y.D. Reijmer, A. ter Telgte, H.J. Kuijf , A. Leemans , \& P.R. Luijten , ... Utrecht Vascular Cognitive Impairment Study Group. (2015). Hippocampal disconnection in early Alzheimer's disease: A 7 tesla MRI study. Journal of Alzheimer's Disease, 45(4), 1247-1256. doi: $10.3233 /$ jad-142994

J.L. Woodard, M. Seidenberg , K.A. Nielson, P. Antuono , L. Guidotti , S. Durgerian, \& S.M. Rao (2009). Semantic memory activation in amnestic mild cognitive impairment. Brain, 132(Pt 8), 2068-2078. doi: 10.1093/brain/awp157

J.L. Woodard, M. Seidenberg , K.A. Nielson, J.C. Smith , P. Antuono, S. Durgerian , \& S.M. Rao (2010). Prediction of cognitive decline in healthy older adults using fMRI. Journal of Alzheimer's Disease, 21, 871-885.

J.L. Woodard, M.A. Sugarman, K.A. Nielson, J.C. Smith, M. Seidenberg, S. Durgerian , \& S.M. Rao (2012). Lifestyle and genetic contributions to cognitive decline and hippocampal structure and function in healthy aging. Current Alzheimer's Research, 9(4), 436-446.

H. Yasmin , Y. Nakata, S. Aoki , O. Abe , N. Sato , K. Nemoto , \& K. Ohtomo (2008). Diffusion abnormalities of the uncinate fasciculus in Alzheimer's disease: Diffusion tensor tract-specific analysis using a new method to measure the core of the tract. Neuroradiology, 50(4), 293-299. doi: 10.1007/s00234-007-0353-7

J.A. Yesavage, T.L. Brink, T.L. Rose, O. Lum , V. Huang , \& M. Adey (1983). Development and validation of a geriatric depression screening scale: A preliminary report. Journal of Psychiatry Research, 17, 37-49.

Y. Zhang , N. Schuff , G.H. Jahng , W. Bayne, S. Mori , L. Schad , \& M.W. Weiner (2007). Diffusion tensor imaging of cingulum fibers in mild cognitive impairment and Alzheimer disease. Neurology, 68(1), 13-19. doi: 10.1212/01.wnl.0000250326.77323.01

L. Zhuang , P.S. Sachdev, J.N. Trollor, N.A. Kochan , S. Reppermund, H. Brodaty , \& W. Wen (2012). Microstructural white matter changes in cognitively normal individuals at risk of amnestic MCI. Neurology, 79(8), 748-754. doi: 10.1212/WNL.0b013e3182661f4d

L. Zhuang , P.S. Sachdev, J.N. Trollor, S. Reppermund, N.A. Kochan , H. Brodaty, \& W. Wen (2013). Microstructural white matter changes, not hippocampal atrophy, detect early amnestic mild cognitive impairment. PLoS One, 8(3), e58887. doi: 10.1371/journal.pone.0058887 